\title{
Quantum noise limits for nonlinear, phase-invariant amplifiers
}

\author{
Dmitri Kouznetsov* \\ Centro de Instrumentos, Universidad Nacional Autónoma de México, Ap.70-186, \\ Cd. Universitaria, 04510 D. F., Mexico, and Lebedev Physics Institute, Leninsky 54, 117924 \\ Russia \\ Roberto Ortega \\ Centro de Instrumentos, Universidad Nacional Autónoma de México, Ap.70-186, \\ Cd. Universitaria, 04510 D. F., Mexico \\ Daniel Rohrlich ${ }^{\dagger}$ \\ School of Physics and Astronomy, Tel-Aviv University, Ramat-Aviv 69978 Tel-Aviv, Israel
}

(August 16, 2018)

\begin{abstract}
Any quantum device that amplifies coherent states of a field while preserving their phase generates noise. A nonlinear, phase-invariant amplifier may generate less noise, over a range of input field strengths, than any linear amplifier with the same amplification. We present explicit examples of such nonlinear amplifiers, and derive lower bounds on the noise generated by a nonlinear, phase-invariant quantum amplifier.

3.65.Bz, 42.50.Ar, 42.50.Lc, 42.65.Ky
\end{abstract}

Typeset using REVTEX

*E-mail: kusnecov@aleph.cinstrum.unam.mx

$\dagger$ †-mail: daniel@vm.tau.ac.il 
Any quantum device that amplifies a field while preserving its phase generates noise. Noise is unavoidable since the phase and number operators for modes of a quantum field do not commute. Two values characterize a phase-preserving or phase-invariant [1] amplifier: the amplification coefficient $G=\langle A\rangle /\langle a\rangle$, and the noise $D=\left\langle A^{\dagger} A\right\rangle-\left\langle A^{\dagger}\right\rangle\langle A\rangle$. Here we focus on a single field mode, for simplicity: $a$ and $A$ denote the field mode before and after amplification, respectively. We take expectation values in a coherent state $|\alpha\rangle$; since $a|\alpha\rangle=\alpha|\alpha\rangle$, the noise in the unamplified field is zero. For a linear quantum amplifier, $G$ is a constant, independent of the initial state, and the minimal noise is well known 2 201: $D \geq|G|^{2}-1$ for $|G| \geq 1$. For a nonlinear amplifier, $G$ depends on $x \equiv \alpha^{*} \alpha$ (but not on the phase of $\alpha$ ). In this Letter, we derive lower limits for the quantum noise in nonlinear quantum amplifiers, and demonstrate that a nonlinear amplifier may generate less noise, for the same amplification, than any linear amplifier, over a range of input field strengths.

What makes an amplifier a quantum amplifier is that the mode operators before and after amplification must be related by a unitary transformation, $A=U^{\dagger} a U$. Thus, $\left[a, a^{\dagger}\right]=1$ implies [2] that $\left[A, A^{\dagger}\right]=1$. In principle, $U$ could depend on the field operators $a$ and $a^{\dagger}$ alone. However, then there will be no amplification. If $U$ depends on $a$ and $a^{\dagger}$ alone, then phase invariance requires it to depend only on $a^{\dagger} a$; the noise, $\left\langle U^{\dagger} a^{\dagger} a U\right\rangle-\left\langle U^{\dagger} a^{\dagger} U\right\rangle\left\langle U^{\dagger} a U\right\rangle=$ $|\alpha|^{2}-|\alpha G(x)|^{2}$, cannot be negative, so $|G| \leq 1$. When $|G(x)|=1$ there is no noise, but the amplifier does not amplify. We must introduce operators for the amplifier, and these induce noise. The choice $U=\exp (-i H t)$, with $H=i\left(a^{\dagger} b^{\dagger}-a b\right)$, leads to a linear amplifier. (The amplifier degree of freedom $b$ obeys $\left[b, b^{\dagger}\right]=1$, and $t$ is real.) We find $A=U^{\dagger} a U=a \cosh t+b^{\dagger} \sinh t$; for an amplifier prepared in the ground state $G=\cosh t$, and the noise $D=\sinh ^{2} t$ saturates the bound $D \leq|G|^{2}-1$ for linear amplifiers.

For nonlinear amplifiers, we have no general expression for $U$ and $A$ in terms of field and amplifier degrees of freedom. Nevertheless, we have the following lower bounds for the noise of a nonlinear, phase-invariant quantum amplifier:

Theorem. Let $D=\left\langle\alpha\left|A^{\dagger} A\right| \alpha\right\rangle-\left\langle\alpha\left|U^{\dagger} A^{\dagger} U\right| \alpha\right\rangle\left\langle\alpha\left|U^{\dagger} A U\right| \alpha\right\rangle$, and 


$$
E(x)=\sum_{n=1}^{\infty} \frac{x^{n+1}}{n !}\left|\frac{\mathrm{d}^{n}}{\mathrm{~d} x^{n}} G(x)\right|^{2}, F(x)=\sum_{n=1}^{\infty} \frac{x^{n-1}}{n !}\left|\frac{\mathrm{d}^{n}}{\mathrm{~d} x^{n}}(x G(x))\right|^{2}-1 ;
$$

Then $D \geq E(x)$ and $D \geq F(x)$.

Proof. Define the set of states $\left|\alpha^{(n)}\right\rangle$

$$
\left|\alpha^{(n)}\right\rangle=\frac{\left(a^{\dagger}-\alpha^{*}\right)^{n}}{\sqrt{n !}}|\alpha\rangle
$$

where $\left\langle\alpha^{(m)} \mid \alpha^{(n)}\right\rangle=\delta_{m n}$ and $\left|\alpha^{(0)}\right\rangle=|\alpha\rangle$. Differentiating the expansion

$$
|\alpha\rangle=e^{-\alpha^{*} \alpha / 2} \sum_{n=0}^{\infty} \frac{\left(\alpha a^{\dagger}\right)^{n}}{n !}|0\rangle
$$

we obtain

$$
\frac{\partial}{\partial \alpha}|\alpha\rangle=-\frac{\alpha^{*}}{2}|\alpha\rangle+a^{\dagger}|\alpha\rangle, \frac{\partial}{\partial \alpha^{*}}|\alpha\rangle=-\frac{\alpha}{2}|\alpha\rangle .
$$

Note that $A=U^{\dagger} a U$ does not depend on $\alpha$, since the amplifier cannot anticipate the input state. Thus, Eqs. (2) imply

$$
\frac{1}{\sqrt{n !}} \frac{\partial^{n}}{\partial \alpha^{n}}(\alpha G)=\left\langle\alpha|A| \alpha^{(n)}\right\rangle, \frac{1}{\sqrt{n !}} \frac{\partial^{n}}{\partial \alpha^{n}}\left(\alpha^{*} G^{*}\right)=\left\langle\alpha\left|A^{\dagger}\right| \alpha^{(n)}\right\rangle .
$$

Consider the identity operator $I_{0}$ in the Hilbert space of states of the field:

$$
I_{0}=\sum_{n=0}^{\infty}\left|\alpha^{(n)}\right\rangle\left\langle\alpha^{(n)}\right|
$$

$I_{0}$ is only part of the identity operator $I$ in the Hilbert space of states of the field and amplifier:

$$
I=\sum_{m, n=0}^{\infty}\left|m, \alpha^{(n)}\right\rangle\left\langle m, \alpha^{(n)}\right|
$$

The index $m$ refers to the states of the amplifier. Without loss of generality, we may identify $I_{0}$ with the $m=0$ term in $I$, where $m=0$ represents the initial state of the amplifier. Inserting $I$ into the expectation value $\left\langle\alpha\left|A^{\dagger} A\right| \alpha\right\rangle$ - where we identify $|\alpha\rangle$ with $|0, \alpha\rangle$ - we obtain

$$
\left\langle\alpha\left|A^{\dagger} A\right| \alpha\right\rangle=\left\langle\alpha\left|A^{\dagger} I A\right| \alpha\right\rangle \geq\left\langle\alpha\left|A^{\dagger} I_{0} A\right| \alpha\right\rangle
$$




$$
D \geq\left\langle\alpha\left|A^{\dagger} I_{0} A\right| \alpha\right\rangle-|\alpha G|^{2}=\sum_{n=1}^{\infty} \frac{1}{n !}\left|\frac{\partial^{n}}{\partial \alpha^{n}}\left(\alpha^{*} G^{*}\right)\right|^{2}=E(x) .
$$

On the other hand, since $A^{\dagger} A=A A^{\dagger}-1$ and $\left\langle\alpha\left|A I A^{\dagger}\right| \alpha\right\rangle \geq\left\langle\alpha\left|A I_{0} A^{\dagger}\right| \alpha\right\rangle$, we have

$$
D \geq\left\langle\alpha\left|A I_{0} A^{\dagger}\right| \alpha\right\rangle-1-|\alpha G|^{2}=\sum_{n=1}^{\infty} \frac{1}{n !}\left|\frac{\partial^{n}}{\partial \alpha^{n}}(\alpha G)\right|^{2}-1=F(x) .
$$

(End of proof)

Both $E(x)$ and $F(x)$ are lower bounds on the noise of a nonlinear, phase-invariant quantum amplifier. When $G$ is constant, $F(x)$ reduces to the bound for linear amplifiers, $|G|^{2}-1$. But both bounds can be less than $|G(x)|^{2}-1$, for a range of values of $x$, if $|G(x)|$ decreases with $x$. $(|G(x)|$ decreasing with $x$ describes amplifier saturation.) We have not yet shown that, for any given $G(x)$, a nonlinear amplifier can realize these lower bounds. A class of amplifiers which do realize the lower bound $F(x)$ have a linear amplification followed by a nonlinear refraction depending only on $a^{\dagger} a$ :

$$
A=e^{i t H}\left[G_{0} a+\left(\left|G_{0}\right|^{2}-1\right)^{1 / 2} b^{\dagger}\right] e^{-i t H}
$$

where $t$ is real. For example, let $H=a^{\dagger} a\left(a^{\dagger} a-1\right) / 2$. We find (using Eq. (3)) that $G(x)=$ $G_{0} \exp (-q x)$, and $D=\left|G_{0}\right|^{2}\left[1+x-x \exp \left(-|q|^{2} x\right)\right]-1$, where $q=1-e^{-i t}$. The amplifier realizes the lower bound $F(x)=D$, while $E(x)=D-1+\left|G_{0}\right|^{2}$. For this class of amplifiers, the linear lower bound $D \geq|G(x)|^{2}-1$ also holds. It is broken in the next example.

The resonant interaction of $N$ identical two-level atoms with a single-mode field can be described [5] by the Hamiltonian $H=i a b^{\dagger}-i a^{\dagger} b$, where $b=\sum_{k=1}^{N} b_{k}, b_{k} b_{j}=(1-$ $\left.\delta_{j k}\right) b_{j} b_{k}, \quad b_{k}^{\dagger} b_{j}=b_{j} b_{k}^{\dagger}$ for $j \neq k$, and $b_{k}^{\dagger} b_{k}+b_{k} b_{k}^{\dagger}=1$. ( $b_{k}^{\dagger}$ is the operator for exciting the $k$-th atom.) The evolution operator $\exp (-i H t)$ defines the transformation $U$. Let all atoms be excited in the initial state of the amplifier. In this case $G$ is real. For $N=10$ and a given value of $t$, let $G_{t}$ denote the amplification factor and $D_{t}$ the noise. We compute the evolution from the initial state numerically. Fig. 1 shows the coefficient $g=G_{t}^{2}$ and the noise $D_{t}$ versus $t$ at input intensity $x=1$. The noise $D_{t}$ oscillates quasiperiodically while $G_{t}$ has "revivals". Fig. 2, a plot of $D_{t}(1)$ versus $\left|G_{t}(1)\right|^{2}$, shows that the linear relation 
$D \geq|G|^{2}-1$ is valid only at small $t$ (for $G_{t}^{2} \lesssim 2$ ), where the amplifier is linear, and violated at larger $x$. Fig. 3 compares $D,|G|^{2}-1, E$, and $F$ as functions of $x$, while Fig. 6 is a plot of the three bounds versus $|G(x)|^{2}$, for $t=0.5$. The nonlinear amplifier beats the linear limit, while the lower limits of the Theorem hold.

The final example involves a small nonlinear perturbation on a linear amplifier. We define

$$
A=C a+S b^{\dagger}-\epsilon\left[a^{\dagger} a^{2}-(C / S) a^{2} b+(2 C / S) a^{\dagger} a b^{\dagger}\right]
$$

where $C \equiv \cosh t, S \equiv \sinh t$, and $\left[a, a^{\dagger}\right]=1=\left[b, b^{\dagger}\right],\left[a, b^{\dagger}\right]=0=[a, b]$. Let $\epsilon$ be small and positive. We find $\left[A, A^{\dagger}\right]=1$ to order $\epsilon$, and $\langle A\rangle=(C-\epsilon x) \alpha$, i.e. $G=C-\epsilon x$. The noise is $D=C^{2}-1-4 C x \epsilon<G^{2}-1$, violating the linear limit to first order in $\epsilon$. It saturates the bound $F(x)$, while $E(x)=0$ to this order.

In summary, we have obtained lower bounds on the irreducible noise of a nonlinear, phasepreserving quantum amplifier, in terms of the amplification factor $G$ and its derivatives. For $G$ constant, the amplifier is linear, and the bound $F$ reproduces the linear bound. For $G$ not a constant, however, the nonlinear bound can undercut the linear bound. Explicit examples confirm that the noise of a nonlinear amplifier can be less than that of an ideal linear amplifier with the same amplification coefficient, over a range of input field strengths. However, we have not determined in general which functions $G(x)$ correspond to nonlinear amplifiers, nor whether the lower bounds can be realized.

\section{ACKNOWLEDGMENTS}

The work of D. K. was partially supported by CONACyT of Mexico. D. R. thanks the Ticho Fund for support. 


\section{REFERENCES}

[1] The term 'phase-preserving' is used for linear amplifiers when $\langle A\rangle$ and $\langle a\rangle$ have the same phase; without loss of generality $G$ may be taken real, and independent of the phase of $\alpha$. Applied to nonlinear amplifiers, however, the term may be ambiguous: $G$ could depend on the phase of $\alpha$ but in such a way that $\langle A\rangle$ and $\langle a\rangle$ have the same phase. For clarity, we call an amplifier 'phase-invariant' when $G$ depends only on $x \equiv \alpha^{*} \alpha$, although $G$ need not be real.

[2] H. A. Haus and J. A. Mullen, Phys. Rev. 128, 2407 (1962).

[3] C. M. Caves, Phys. Rev. D26, 1817 (1982).

[4] Y. Yamamoto and H. A. Haus, Rev. Mod. Phys. 58, 1001 (1986).

[5] M. Tavis and F. M. Cummings. Phys. Rev. 188, 692 (1969). 


\section{FIGURES}

FIG. 1. Amplification coefficient $g=G_{t}^{2}$ and noise $D_{t}$ versus $t$ at $x=1$ for the Cummings-Tavis amplifier with $N=10$ atoms. At $t=0, G^{2}=1$ (thick line) and $D=0$ (thin line).

FIG. 2. The noise $D_{t}$ versus $G_{t}^{2}$ under the same conditions.

FIG. 3. The noise $D$ (thick curve), linear lower bound $G^{2}-1$ (thin curve), and lower bounds $E$ (dotted curve) and $F$ (dashed curve) versus $x$ for the Cummings-Tavis amplifier at $N=10$, $t=0.5$.

FIG. 4. The noise $D$ and all bounds versus $G^{2}$ under the same conditions. 


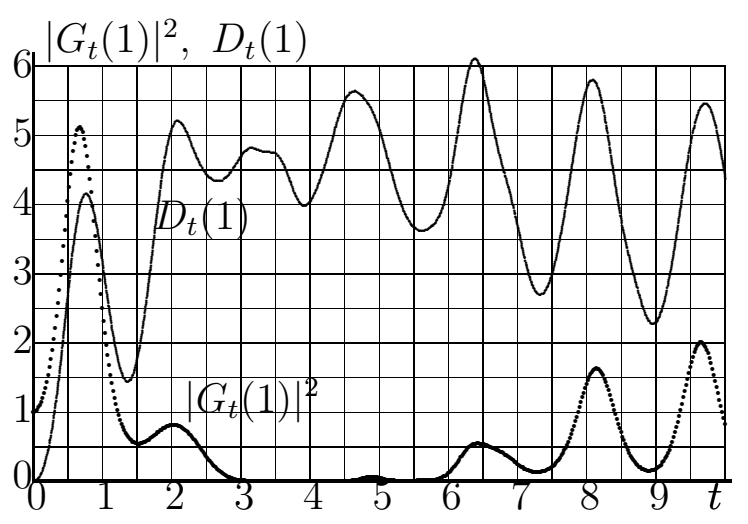

FIG. 1. Amplification coefficient $g=G_{t}^{2}$ and noise $D_{t}$ versus $t$ at $x=1$ for the CummingsTavis amplifier with $N=10$ atoms. At $t=0, G^{2}=1$ (thick dotted line) and $D=0$ (thin line). 


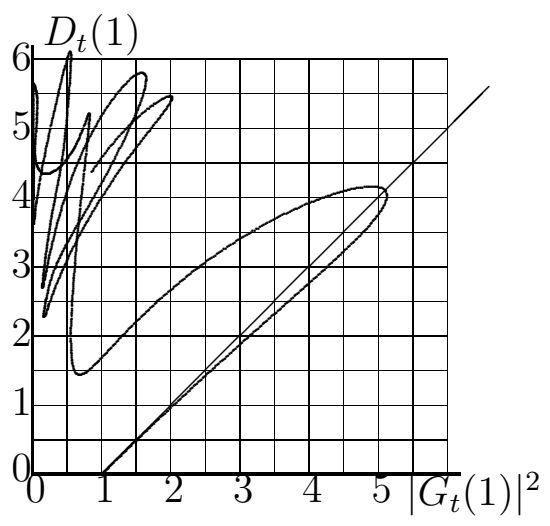

FIG. 2. The noise $D_{t}$ versus $G_{t}^{2}$ under the same conditions. 




FIG. 3. The noise $D$ (thick curve), linear lower bound $G^{2}-1$ (thin curve), and lower bounds $E$ (dotted curve) and $F$ (dashed curve), versus $x$ for the Cummings-Tavis amplifier at $N=10, t=0.5$. 


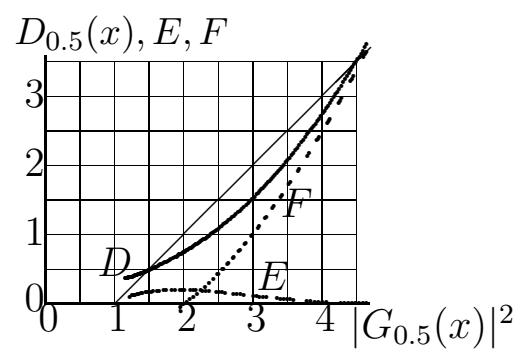

FIG. 4. The noise $D$ and all bounds versus $|G|^{2}$ under the same conditions. 